\title{
Caracterización molecular y resistencia antimicrobiana de aislamientos de Clostridium perfringens de diferentes orígenes en Costa Rica
}

\author{
María del Mar Gamboa-Coronado*, Silvia Mau-Inchaustegui \& Evelyn Rodríguez-Cavallini \\ Laboratorio de Investigación en Bacteriología Anaerobia y Centro de Investigación en Enfermedades Tropicales, \\ Facultad de Microbiología, Universidad de Costa Rica, Ciudad Universitaria Rodrigo Facio, San Pedro de Montes de \\ Oca 2060, San José, Costa Rica; maria.gamboa@ucr.ac.cr, silvyamau@gmail.com, evelyn.rodriguez@ucr.ac.cr \\ * Correspondencia
}

\author{
Recibido 25-X-2010. ～Corregido 20-II-2011． Aceptado 23-III-2011.
}

\begin{abstract}
Molecular characterization and antimicrobial resistance of Clostridium perfringens isolates of different origins from Costa Rica. Clostridium perfringens, a Gram positive, spore-forming anaerobe, is widely distributed in nature. Based upon their production of four major toxins $\alpha, \beta, \varepsilon$ and $\mathrm{\iota}, C$. perfringens is classified into five toxinotypes (A-E). Some strains produce an enterotoxin (CPE), encoded by the cpe gene, which causes diarrhea in humans and some animals. C. perfringens strains that had been previously isolated and been kept at $-80^{\circ} \mathrm{C}$ were analyzed for the presence of toxin genes and for antimicrobial resistance: 20 from soils, 20 from animal, 20 from human origin and 21 from food non related to outbreaks. According to PCR results, all strains were classified as $C$.perfringens type A, since only $\alpha$ toxin gene was detected, while cpe was detected in two strains (2.5\%) isolated from food, as it has been described in other world regions. Antibiotic resistance to at least one antibiotic was detected in $44 \%$ of the strains, $41 \%$ was resistant to clindamycin, $25 \%$ to chloramphenicol, $22 \%$ to penicillin and $20 \%$ to metronidazole. Soils strains showed the highest resistance percentages to almost all antibiotics. Multiresistance (to three or more antibiotic groups) was detected in the strains from soil $(40 \%)$, human origin (30\%), food (14\%) and animal origin (5\%). The high resistance rates found may be explained by the widespread use of antimicrobials as growth promoters in plants and animals; also these resistant strains may act as reservoir of resistance genes that may be transferred between bacteria in different environments. Rev. Biol. Trop. 59 (4): 1479-1485. Epub 2011 December 01.
\end{abstract}

Key words: Clostridium perfringens, enterotoxin, toxinotypes, antimicrobial resistance.

Clostridium perfringens es un bacilo Gram positivo, esporulado, anaerobio, que con frecuencia se encuentra en suelos y aguas negras y en tracto gastrointestinal de animales y humanos como miembro de su microbiota intestinal (Petit et al.1999). Es responsable de una variedad de enfermedades en seres humanos y animales (Labbe 2001). Su patogenicidad se asocia con la producción de muchas toxinas, de las cuales las principales son la alfa $(\alpha)$, beta $(\beta)$, épsilon $(\varepsilon)$ e iota ( $)$. $C$. perfringens se clasifica en cinco toxinotipos (A-E) de acuerdo con los tipos de toxina que produzca; el tipo A produce solo toxina $\alpha$, el B produce $\alpha, \beta$ y $\varepsilon$, el
C produce $\alpha$ y $\beta$, el D produce $\alpha$ y $\varepsilon$, el tipo $\mathrm{E}$ produce $\alpha$ y ı (Petit et al. 1999). Cada toxinotipo se asocia con una enfermedad en particular, por lo que su determinación es importante para el diagnóstico y el estudio epidemiológico de los cuadros clínicos (Petit et al. 1999).

Además de estas toxinas, algunas cepas de C. perfringens, que generalmente pertenecen al tipo A, producen una enterotoxina (CPE) que causa diarrea en seres humanos y en algunos animales y que es codificada por el gen cpe (Baums et al. 2004). Tradicionalmente la clasificación de las cepas de C. perfringens se ha realizado por seroneutralización utilizando 
ratones o cobayos (Heinkinheimo \& Korkeala 2005). Sin embargo, estos métodos son costosos, toman tiempo y son cuestionados éticamente por utilizar animales, por lo que en los últimos años han sido reemplazados por métodos moleculares (Baums et al. 2004), a pesar de que la demostración de los genes que codifican por las diferentes toxinas no implica necesariamente, que la proteína codificada esté siendo producida (Van Asten et al. 2009).

La producción de la CPE por parte de $C$. perfringens solo ocurre durante la esporulación y debido a que esta bacteria esporula mal en los medios de cultivo, su detección se dificulta (Petit et al. 1999). El uso de técnicas moleculares ha permitido, por lo tanto, solventar estos inconvenientes y poder determinar cuáles cepas son las que tienen el gen (Baums et al. 2004).

Por otra parte, el conocimiento del perfil de sensibilidad a los antibióticos de $C$. perfringens de diferentes orígenes, incluyendo suelos, alimentos, animales y seres humanos, puede contribuir a una utilización más racional de los antibióticos tanto para tratamiento clínico, como para usos agropecuarios, y la detección de cepas con resistencia antimicrobiana podría alertar sobre una posible transferencia de genes entre bacterias de diferentes orígenes.

En Costa Rica, C. perfringens ha sido aislado de numerosos ambientes: del $88 \%$ de suelos aledaños a plantas procesadoras de carne, del $93 \%$ de contenidos intestinales de ganado, del $55 \%$ de la carne en canal, del $75 \%$ de carnes molidas, del $36 \%$ de carnes en trozos para la venta al detalle y de $3 \%$ de carnes cocidas mantenidas en baños termo-regulables (Rodríguez et al. 2002). Sin embargo, en ausencia de conocimientos más específicos, este estudio aborda una caracterización fenotípica y genotípica de cepas costarricenses de diferentes orígenes, con el fin de contribuir al conocimiento de su distribución epidemiológica.

\section{MATERIALES Y MÉTODOS}

Cepas de $C$. perfringens: De la colección de bacterias anaerobias del Laboratorio de Investigación en Bacteriología Anaerobia
(LIBA), Universidad de Costa Rica se analizaron 81 cepas de $C$. perfringens: 20 de suelos, 20 de origen animal, 20 de origen humano y 21 de alimentos cocidos no relacionados con brotes alimentarios. Todas habían sido aisladas previamente siguiendo las metodologías estándar y se habían conservado a $-80^{\circ} \mathrm{C}$ (Rodríguez et al. 2002). Cada una de las cepas se recuperó en medio carne picada PRAS (Holdeman et al. 1977), el cual se incubó a $35^{\circ} \mathrm{C}$ por $24-48 \mathrm{hr}$ y posteriormente se inoculó en agar sangre para verificar su pureza.

Aislamiento del ADN total: El ADN se purificó según la metodología de Murray \& Thompson (1980). Brevemente, cada cepa se creció en medio carne picada PRAS a $35^{\circ} \mathrm{C}$ por 24hr. Las bacterias por se concentraron centrifugación a $6000 \mathrm{~g}$ por $10 \mathrm{~min}$, se resuspendieron en $567 \mu \mathrm{L}$ de buffer TE $(10 \mathrm{mM}$ Tris- $\mathrm{HCl}$, $1 \mathrm{mM}$ EDTA, pH 8.0) y se incubaron a $37^{\circ} \mathrm{C}$ por $1 \mathrm{hr}$ después de añadir $30 \mu \mathrm{L}$ de duodecil sulfato de sodio al $10 \%$ y $3 \mu \mathrm{L}$ de proteinasa $\mathrm{K}(20 \mathrm{mg} /$ $\mathrm{mL}$ en agua destilada). Luego se agregó $100 \mu \mathrm{L}$ de solución de $\mathrm{NaCl} 5 \mathrm{M}$ y $80 \mu \mathrm{L}$ de solución CTAB-NaCl (cetiltrimetil bromuro de amonio al $10 \%$ en $\mathrm{NaCl}(7 \mathrm{M})$ y se incubó a $65^{\circ} \mathrm{C}$ por $10 \mathrm{~min}$. Con solución de fenol-cloroformo-alcohol isoamílico (25:24:1) se hizo una extracción de ADN y se precipitació con etanol absoluto por incubación a $-20^{\circ} \mathrm{C}$ toda la noche; luego se centrifugó a $12000 \mathrm{~g}$ por $20 \mathrm{~min}$, se lavó con alcohol de $70 \%$ y se resuspendió en $100 \mu \mathrm{L}$ de buffer TE, después de que fue secado a $37^{\circ} \mathrm{C}$. El ADN se mantuvo a $-20^{\circ} \mathrm{C}$ hasta su uso.

\section{Caracterización molecular por PCR:} Para detectar las toxinas alfa $(\alpha)$, beta $(\beta)$ y épsilon $(\varepsilon)$ se empleó un PCR múltiple y los primers diseñados por Yoo et al. (1997). La mezcla contenía $12.5 \mu \mathrm{L}$ de Master Mix (Fermentas $\left.{ }^{\circledR}\right), 7.5 \mu \mathrm{L}$ de agua para PCR, $1.0 \mu \mathrm{L}$ de cada uno de los 5 primers $(10 \mu \mathrm{M})$ y $1.0 \mu \mathrm{L}$ de ADN. El Master Mix (2X) contenía Taq polimerasa $(0.05 \mathrm{U} / \mu \mathrm{L}), \mathrm{MgCl}_{2}(4 \mathrm{mM})$ y una mezcla de dNTPs $(0.4 \mathrm{mM}$ de cada uno). Para la amplificación del $\mathrm{ADN}$ se incubó $5 \mathrm{~min}$ a $94^{\circ} \mathrm{C}$, seguido de 30 ciclos de $1 \mathrm{~min}$ a $55^{\circ} \mathrm{C}$, 
$1 \mathrm{~min}$ a $72^{\circ} \mathrm{C}$ y $1 \mathrm{~min}$ a $94^{\circ} \mathrm{C}$, y un paso final de extensión de $5 \mathrm{~min}$ a $72^{\circ} \mathrm{C}$. Como controles se utilizaron las cepas $C$. perfringens tipo A ATCC 13124 y $C$. perfringens tipo B ATCC 3226. El análisis de los productos de amplificación se hizo por electroforesis en gel de agarosa al $2 \%$.

Para detectar el gen cpe se siguió la metodología así como los primers P145 y P146 descritos por Fach \& Popoff (1997), los cuales amplifican un fragmento de 426pb; se empleó la mezcla para PCR Master Mix (2X). El programa de amplificación consistió de $5 \mathrm{~min}$ a $94^{\circ} \mathrm{C}$, seguido de 30 ciclos de 30 s a $94^{\circ} \mathrm{C}, 30$ s a $55^{\circ} \mathrm{C}$ y $30 \mathrm{~s}$ a $72^{\circ} \mathrm{C}$ y por último se realizó una extensión de $72^{\circ} \mathrm{C}$ por $10 \mathrm{~min}$. Como controles se utilizaron las cepas $C$. perfringens LIBA 89 $\left(c p e^{+}\right)$y C. perfringens ATCC 13124 (cpe $e^{-}$.

Pruebas de sensibilidad a los antibióticos: La galería comercial ATB ANA de la casa Biomeriux ${ }^{\circledR}$ se utilizó siguiendo todas las recomendaciones del fabricante para su inoculación, incubación e interpretación.

\section{RESULTADOS}

Las 81 cepas de $C$. perfringens de diferentes orígenes: animales, humanos, alimentos cocidos y suelos, fueron clasificadas como $C$. perfringens tipo $\mathrm{A}$, debido a que sólo se les detectó el gen de la toxina alfa $(\alpha)$, mientras que el gen de la enterotoxina (cpe) se encontró solamente en dos de las 81 cepas estudiadas (2.5\%), las cuales habían sido aisladas de alimentos cocidos.

El 44\% de todas las cepas fue resistente a algún antibiótico; en el Cuadro 1 se muestran los resultados de resistencia a los antibióticos que presentaron las cepas de $C$. perfringens. Respecto a la clindamicina el $41 \%$ fue resistente: $70 \%$ de las cepas de origen humano, $40 \%$ de las de suelo, $29 \%$ de las de alimentos y $25 \%$ de las de origen animal. Además, el $20 \%$ de todas las cepas fue resistente al metronidazol: las del suelo presentaron el mayor porcentaje de resistencia $(50 \%)$, seguidas de las cepas de origen animal y de alimentos (15\% y $14 \%$,

\section{CUADRO 1}

Porcentaje de resistencia a los antimicrobianos de cepas costarricenses de Clostridium perfringens aisladas de animales $(n=20)$, humanos $(n=20)$, alimentos $(n=21)$ y suelos $(n=20)$

TABLE 1

Antimicrobial resistance percentage of Costa Rican Clostridium perfringens strains isolated from animals $(\mathrm{n}=20)$, humans $(n=20)$, food $(n=21)$ and soils $(n=20)$

\begin{tabular}{lccccc} 
& $\begin{array}{c}\text { Cepas de } \\
\text { animales } \%\end{array}$ & $\begin{array}{c}\text { Cepas de } \\
\text { humanos } \%\end{array}$ & $\begin{array}{c}\text { Cepas de } \\
\text { alimentos \% }\end{array}$ & $\begin{array}{c}\text { Cepas de } \\
\text { suelos \% }\end{array}$ & Total \% \\
Clindamicina & 25 & 70 & 29 & 40 & 41 \\
Metronidazol & 15 & 0 & 14 & 50 & 20 \\
Cloranfenicol & 20 & 30 & 24 & 25 & 25 \\
Penicilina & 15 & 20 & 14 & 40 & 22 \\
Amoxicilina & 0 & 0 & 0 & 10 & 2 \\
Amoxicilina / ác. clavulánico & 0 & 0 & 0 & 0 & 0 \\
Piperacilina & 0 & 5 & 0 & 10 & 4 \\
Piperacilina / Tazobactam & 0 & 0 & 0 & 5 & 0 \\
Ticarcilina & 0 & 0 & 0 & 5 & 1 \\
Ticarcilina / ác. clavulánico & 0 & 0 & 0 & 20 & 1 \\
Cefoxitina & 5 & 0 & 5 & 20 & 7 \\
Cefotetán & 5 & 0 & 5 & 0 & 7 \\
Imipinem & 0 & 0 & 0 & 0 & 0 \\
\hline
\end{tabular}


respectivamente), mientras que ninguna de las de origen humano fue resistente. Con respecto al cloranfenicol, el 25\% de todas las cepas fue resistente, y según el origen de las cepas, la resistencia osciló entre el 20 y el $30 \%$. El $22 \%$ de las cepas fue resistente a penicilina, el mayor porcentaje de resistencia lo presentó las cepas provenientes de suelo (40\%), mientras que solo el $15 \%$ de las cepas de origen animal y el $20 \%$ de origen humano fue resistente.

En general, al comparar las cepas provenientes de diversos orígenes, las de suelos presentaron los mayores porcentajes de resistencia a casi todos los antibióticos (Cuadro 1). Incluso algunos antibióticos (cefoxitina, cefotetán, piperacilina, amoxicilina, ticarcilina y ticarcilina con ácido clavulánico) a los cuales no se les detectó resistencia en las cepas de animales, de humanos y de alimentos, sí se les detectó en las de cepas de suelo (del 5 al 20\%, Cuadro 1).

El $40 \%$ de las cepas de suelo fue multiresistente (a tres o más grupos de antibióticos), incluso tres cepas fueron resistentes a cinco de los siete grupos de antibióticos evaluados (penicilina, cefalosporinas, clindamicina, cloranfenicol y metronidazol). Aunque la multiresistencia de las cepas de origen humano fue del $30 \%$, solo una cepa fue resistente a cuatro de los siete grupos de antibióticos evaluados (penicilina, clindamicina, cloranfenicol y metronidazol). La multiresistencia de las cepas de alimentos y de origen animal fue mucho menor ( $14 \%$ y $5 \%$, respectivamente), y solo un aislamiento de cada uno de estos orígenes fue resistente a cuatro de los siete grupos de antibióticos.

\section{DISCUSIÓN}

En el presente trabajo se demostró una distribución ecológica de $C$. perfringens similar a la de otras regiones, con un predominio del toxinotipo A (Petit et al. 1999), tanto en sedimentos marinos como suelos (Fach \& Popoff 1997); Li et al. (2007) informaron que el $98.8 \%$ de las cepas de suelos en Estados Unidos eran tipo A. Este toxinotipo predomina en la flora intestinal de muchos animales, incluyendo el ser humano (Heikinheimo \& Korkeala 2005, Heikinheimo et al. 2006) y es responsable de muchos cuadros clínicos en humanos, tales como gangrena, diarrea asociada a antibióticos (Joshy et al. 2006), así como cuadros gastrointestinales en otros animales (Petit et al. 1999, Baums et al. 2004). Finalmente, numerosos estudios realizados en Estados Unidos y Japón han demostrado que la mayoría de las cepas de $C$. perfringens provenientes de alimentos, relacionados o no con brotes alimentarios, son del tipo A (Lin \& Labbe 2003, Wen \& McClane 2004, Miki et al. 2008) tal como se encontró en este trabajo.

El gen de la enterotoxina (cpe) sólo se encontró en dos de las cepas estudiadas (2.5\%), las cuales habían sido aisladas de alimentos. La frecuencia de este gen en cepas de origen alimentario generalmente es baja, del 0 al $4 \%$ en alimentos de origen animal (Lin \& Labbe 2003, Wen \& McClane 2004, Miki et al. 2008, Rahmati \& Labbe 2008). En contraste con estos bajos porcentajes, Miwa et al. (1998) determinaron que el $17 \%$ de las cepas aisladas de muestras de carne y pollo tenían el gen cpe. En el presente estudio el $10 \%$ de las cepas provenientes de alimentos contenían el gen de la enterotoxina, mientras que previamente en Costa Rica se encontró que el 15\% de cepas de alimentos cocidos eran toxigénicas (Gutiérrez et al. 1999), desconocemos si esto obedece a razones metodológicas o a cambios reales en el tiempo.

La mayoría de los estudios señalan que las cepas enterotoxigénicas son poco frecuentes en diferentes tipos de muestras; en cepas de heces de individuos asintomáticos varían desde 0 hasta 7.5\% (Joshy et al. 2006), en cepas de origen animal desde 0 hasta $22 \%$ (Tschirdewahn et al. 1992, Heikinheimo \& Korkeala 2005) y en cepas de suelos desde 0 hasta $7 \%$ (Kuske et al. 2006, Li et al. 2007). A pesar de que la incidencia de cepas enterotoxigénicas de $C$. perfringens sea baja, éstas representan un riesgo porque pueden dar origen a cuadros clínicos o brotes.

Aunque era de esperar que el porcentaje de cepas toxigénicas fuera bajo, es posible que las 
cepas hayan correspondido, por azar, a cepas no toxigénicas, porque en una misma muestra puede coexistir un pequeño número de células cpe-positivas con un gran número de células cpe-negativas (Miwa et al. 1997, Heikinheimo et al. 2004, Heikinheimo et al. 2006).

En el presente estudio el porcentaje de cepas resistentes a algún antibiótico fue del $44 \%$. Aunque existen pocos estudios sobre el perfil de susceptibilidad a los antimicrobianos de cepas de $C$. perfringens de diferentes orígenes, en Tailandia el $62.7 \%$ de cepas de heces de humanos y de cerdos, alimentos y del ambiente fue resistente a uno o varios antibióticos (Tansuphasiri et al. 2005), lo que indica que en ambos países la resistencia es frecuente y podría explicarse por el uso que se le da a los antibióticos.

La clindamicina es uno de los antibióticos más utilizados para el tratamiento de infecciones por anaerobios, por lo que es preocupante que el $41 \%$ de las cepas fuera resistente, y particularmente alarmante que el $70 \%$ de las cepas de origen humano lo fuera (Cuadro 1). También los otros grupos de cepas presentaron altos porcentajes de resistencia: $40 \%$ de las de suelo, $29 \%$ de las de alimentos y $25 \%$ de las de origen animal (Cuadro 1). Aunque la resistencia a clindamicina de cepas de origen clínico humano es poco frecuente en otras latitudes (Alexander et al. 1995, Citron et al. 2005), los porcentajes de resistencia obtenidos en este estudio sugieren que en Costa Rica podría ser alto, por lo que debería corroborarse con aislamientos de cuadros clínicos.

Respecto al metronidazol, otro de los tratamientos de elección contra los anaerobios, el $20 \%$ de todas las cepas fue resistente, mientras que ninguna de origen humano fue resistente (Cuadro 1). La resistencia a metronidazol no es muy frecuente (Alexander et al. 1995, Citron et al. 2005), pero se ha reportado que en Tailandia y en Costa Rica un 13.5 y un $10.4 \%$, respectivamente, de cepas de origen humano eran resistentes (Tansuphasiri et al. 2005, Camacho et al. 2008).

En un estudio previo con cepas de heces humanas en Costa Rica (Camacho et al. 2008), no se detectó resistencia al cloranfenicol, lo que contrasta con el porcentaje de cepas resistentes de origen humano que se detectó $(30 \%)$ en el presente trabajo. Tampoco se detectaron cepas resistentes en alimentos en Tailandia (Tansuphasiri et al. 2005), aunque en el presente estudio el $24 \%$ de la cepas fue resistente. Estas diferencias podrían deberse a que se emplearon metodologías diferentes en ambos estudios o a que esta resistencia es de reciente aparición en nuestro país. La resistencia de cepas de $C$. perfringens sí ha sido descrita en cepas aisladas de suelo en Grecia (Voidarou et al. 2006), y concuerda con la detección de un $25 \%$ de cepas resistentes provenientes de suelos en el presente estudio.

También se detectó un alto porcentaje de resistencia a la penicilina (22\%), particularmente en las cepas provenientes de suelo (40\%), situación que también fue descrita en un estudio en Grecia (Voidarou et al. 2006). Las diferencias en las prácticas terapéuticas y veterinarias de los países podrían explicar que en el presente estudio el $15 \%$ de las cepas de origen animal y el $20 \%$ de origen humano fueran resistentes a la penicilina, mientras que ninguna de las cepas de intestino de pollos de otro estudio (Silva et al. 2009) y solo el 10\% de las cepas de heces humanas fuera resistente (Tansuphasiri et al. 2005).

En general, los mayores porcentajes de resistencia para casi todos los antibióticos se presentaron en cepas provenientes de suelos, tal como ha sido descrito en Grecia (Voidarou et al. 2006), y esto puede ser un reflejo del uso indiscriminado de antibióticos en la agricultura (Tansuphasiri et al. 2005).

En concordancia con lo anterior, el mayor porcentaje de cepas multiresistentes provenía de suelo (40\%), incluso las cepas resistentes a cinco de los siete grupos de antibióticos evaluados, provenían de suelo. Esta multiresistencia es preocupante por la posibilidad de que estas cepas puedan actuar como un reservorio de genes de resistencia por el uso tan difundido de antimicrobianos como promotores de crecimiento de plantas y animales para el consumo humano. 
Aunque se observó multiresistencia en cepas de origen humano y de alimentos (30 y $14 \%$, respectivamente), ésta fue menor a la observada en Tailandia, donde las cepas de flora intestinal humana y de alimentos presentaron porcentajes más altos de multiresistencia (33.7 y 20\%, respectivamente, Tansuphasiri et al. 2005); esto podría deberse a diferencias en el uso de los antibióticos en ambos países.

La demostración de que el 10\% de las cepas de $C$. perfringens aisladas de alimentos poseen el gen de la enterotoxina refuerza la importancia de investigar los posibles reservorios que puedan favorecer la aparición de brotes de cuadros clínicos causados por esta bacteria. Además el presente estudio ha permitido conocer por primera vez en Costa Rica, el perfil de resistencia a los antibióticos de $C$. perfringens, el patógeno más distribuido en la naturaleza, lo que contribuirá a mejorar el manejo de pacientes con este tipo de infecciones. Finalmente, este estudio puso de manifiesto la amplia distribución de cepas resistentes a los antibióticos a partir de diferentes orígenes, lo que probablemente refleja el uso indiscriminado de antibióticos y plantea la posibilidad de transferencia de esos genes de resistencia entre bacterias de diversos ambientes.

\section{AGRADECIMIENTOS}

Los autores agradecen a Pablo Vargas y Martín Quesada por su ayuda técnica y a la Vicerrectoría de Investigación de la Universidad de Costa Rica por el financiamiento otorgado.

\section{RESUMEN}

Clostridium perfringens es un bacilo Gram positivo, esporulado, anaerobio, ampliamente distribuido en la naturaleza, que produce cuatro toxinas principales $\alpha, \beta, \varepsilon \mathrm{y} \mathrm{t}$, las cuales permiten su clasificación en cinco toxinotipos (A-E). Algunas cepas producen una enterotoxina (CPE), codificada por el gen cpe, que causa diarrea en seres humanos y en algunos animales. La presencia de los genes de estas toxinas y la sensibilidad a los antibióticos se determinó en 81 cepas de $C$. perfringens previamente aisladas y que habían sido mantenidas a $-80^{\circ} \mathrm{C} ; 20$ de suelos, 20 de origen animal, 20 de origen humano y 21 de alimentos cocidos no relacionados con brotes alimentarios. De acuerdo con los resultados de PCR, todas las cepas fueron clasificadas como $C$. perfringens tipo A, debido a que solo se les detectó el gen de la toxina $\alpha$, mientras que el gen de la enterotoxina (cpe) se detectó en dos cepas (2.5\%) aisladas de alimentos, tal como ha sido descrito en otras regiones del mundo. El $44 \%$ de las cepas fue resistente a algún antibiótico; clindamicina (41\%), cloranfenicol (25\%), penicilina (22\%) y metronidazol (20\%). En general, las cepas provenientes de suelos presentaron los mayores porcentajes de resistencia a casi todos los antibióticos. El $40 \%$ de las cepas de suelo presentó multiresistencia (a tres o más grupos de antibióticos), el 30\% de las de origen humano, el $14 \%$ de las de alimentos y el $5 \%$ de las de origen animal. Las altas tasas de resistencia encontradas podrían deberse al amplio uso de antibióticos como promotores de crecimiento de plantas y animales y esas cepas resistentes podrían actuar como reservorio de genes de resistencia que pueden transferirse entre bacterias de diversos ambientes.

Palabras clave: Clostridium perfringens, enterotoxinas, toxinotipos, resistencia antimicrobiana.

\section{REFERENCIAS}

Alexander, C.J., D.M. Citron, J.S. Brazier \& E.J.C. Goldstein. 1995. Identification and antimicrobial resistance patterns of clinical isolates of Clostridium clostridioforme, Clostridium innocuum, and Clostridium ramosum compared with those of clinical isolates of Clostridium perfringens. J. Clin. Microbiol. 33: 3209-3215.

Baums, C.G., U. Schotte, G. Amtsberg \& R. Goethe. 2004. Diagnostic multiplex PCR for toxin genotyping of Clostridium perfringens isolates. Vet. Microbiol. 100: 11-16.

Camacho, N., C. Espinoza, C. Rodríguez \& E. Rodriguez. 2008. Isolates of Clostridium perfringens recovered from Costa Rican patients with antibiotic-associated diarrhoea are mostly enterotoxin-negative and susceptible to first-choice antimicrobials. J. Med. Microbiol. 57: 343-347.

Citron, D.M., Y.Y. Kwok \& M.D. Appleman. 2005. In vitro activity of oritavancin (LY333328), vancomycin, clindamycin, and metronidazole against Clostridium perfringens, Propionibacterium acnes, and anaerobic gram positive cocci. Anaerobe 11: 93-95.

Fach, P. \& M.R. Popoff. 1997. Detection of enterotoxigenic Clostridium perfringens in food and fecal samples with a duplex PCR and the slide latex agglutination test. Appl. Environ. Microbiol. 63: 4232-4236.

Gutiérrez, A., M.M. Gamboa, E. Rodríguez \& M.L. Arias. 1999. Presencia de Clostridium perfringens en 
preparaciones a base de carne en servicios de alimentación del Cantón Central de San José, Costa Rica. Arch. Latinoam. Nutr. 49: 275-278.

Heikinheimo, A. \& H. Korkeala. 2005. Multiplex PCR assay for toxinotyping Clostridium perfringens isolates obtained from Finnish broiler chickens. Lett. Appl. Microbiol. 40: 407-411.

Heikinheimo, A., M. Lindstrom \& H. Korkeala. 2004. Enumeration and isolation of cpe-positive Clostridium perfringens spores from feces. J. Clin. Microbiol. 42: 3992-3997.

Heikinheimo, A., M. Lindstrom, P.E. Granum \& H. Korkeala. 2006. Humans as reservoir for enterotoxingene-carrying Clostridium perfringens type A. Emerg. Infect. Dis. 12: 1724-1729.

Holdeman, L.V., E.P. Cato \& W.E.C Moore. 1977. Anaerobe laboratory manual. Virginia Polytechnic Institute and State University, Blacksburg, Virginia, EEUU.

Joshy, L., R. Chaudhry, B. Dhawan, L. Kumar \& B.K. Das. 2006. Incidence and characterization of Clostridium perfringens isolated from antibiotic-associated diarrhoeal patients: a prospective study in an Indian hospital. J. Hosp. Infect. 63: 323-329.

Kuske, C.R., S.M. Barns, C.C. Grow, L. Merril \& J. Dunbar. 2006. Environmental survey for four pathogenic bacteria and closely related species using phylogenetic and functional genes. J. Forensic Sci. 51: $548-558$

Labbe, R. 2001. Clostridium perfringens, p. 325-330. In F. Pouch-Downes \& K. Ito (eds.). Compendium of methods for the microbiological examination of foods, American Public Health Association, Washington, DC, EEUU.

Li, J., S. Sayeed \& B.A. McClane. 2007. Prevalence of enterotoxigenic Clostridium perfringens isolates in Pittsburgh (Pennsylvania) area soils and home kitchens. Appl. Environ. Microbiol. 73: 7218-7224.

Lin, Y.T. \& R. Labbe. 2003. Enterotoxigenicity and genetic relatedness of Clostridium perfringens isolates from retail foods in the United States. Appl. Environ. Microbiol. 69: 1642-1646.

Miki, Y., K. Miyamoto, I. Kaneko-Hirano, K. Fujiuchi \& S. Akimoto. 2008. Prevalence and characterization of enterotoxin gene-carrying Clostridium perfringens isolates from retail meat products in Japan. Appl. Environ. Microbiol. 74: 5366-5372.

Miwa, N., T. Nishina, S. Kubo \& M. Atsumi. 1997. Most probable number method combined with nested polymerase chain reaction for detection and enumeration of enterotoxigenic Clostridium perfringens in intestinal contents of cattle, pig and chicken. J. Vet. Med. Sci. 59: 89-92.

Miwa, N., T. Nishina, T.S. Kubo, M. Atsumi \& H. Honda. 1998. Amount of enterotoxigenic Clostridium perfringens in meat detected by nested PCR. Int. J. Food Microbiol. 42: 195-200.

Murray, M.G. \& W.F. Thompson. 1980. Rapid isolation of high-molecular weight plant DNA. Nucleic Acids Res. 8: 4321-4325.

Petit, L., M. Gibert \& M.R. Popoff. 1999. Clostridium perfringens: toxinotype and genotype. Trends Microbiol. 104: 104-110.

Rahmati, T. \& R. Labbe. 2008. Levels and toxigencity of Bacillus cereus and Clostridium perfringens from retail seafood. J. Food Prot. 71: 1178-1185.

Rodríguez, E., M.M. Gamboa \& P. Vargas. 2002. Clostridium perfringens en carnes crudas y cocidas y su relación con el ambiente en Costa Rica. Arch. Latin. Nutr. 52: 155-159.

Silva, R.O.S., F.M. Salvarani, N.R.S. Martins, P.S. Pires \& F.C.F. Lobato. 2009. Antimicrobial susceptibility of Clostridium perfringens strains isolated. Braz. J. Microbiol. 40: 262-264.

Tansuphasiri, U., W. Matra \& L. Sangsuk. 2005. Antimicrobial resistance among Clostridium perfringens isolated from various sources in Thailand. SE Asian J. Trop. Med. 36: 954-960.

Tschirdewahn, B., S. Notermans, K. Wernars \& F. Untermann. 1992. The presence of Clostridium perfringens strains in faeces of various animals. Int. J. Food Microbiol. 14: 175-178.

Van Asten, A.J., C.W. Van der Wiel, G. Nikolaou, D.J. Houwers \& A. Grone. 2009. A multiple PCR for toxin of Clostridium perfringens isolates. Vet. Microbiol. 136: 411-4112.

Voidarou, C., S. Kandrelis, D. Vassos, A. Tzora, I. Skoufos, A. Alexopoulos \& E. Bezirtzoglou. 2006. Occurrence of Clostridium perfringens from different cultivated soil. $18^{\text {th }}$ World Congress of Soil Science, Philadelphia, Pennsylvania, EEUU.

Wen, Q. \& B.A. McClane. 2004. Detection of enterotoxigenic Clostridium perfringens type A isolates in American retail foods. Appl. Environ. Microbiol. 70: 2685-2691.

Yoo, H.S., S.U. Le, K.Y. Park \& Y.H. Park. 1997. Molecular typing and epidemiological survey of prevalence of Clostridium perfringens types by multiplex PCR. J. Clin. Microbiol. 35: 228-232. 
